\title{
Kondo-lattice behavior and multiple characteristic temperatures in $\mathrm{CeIr}_{2} \mathrm{Ge}_{2}$
}

\author{
R. Mallik, E. V. Sampathkumaran, and P. L. Paulose \\ Tata Institute of Fundamental Research, Homi Bhabha Road, Colaba, Mumbai 400005, India \\ J. Dumschat and G. Wortmann \\ Universität-GH Paderborn, Fachbereich Physik, D-33095 Paderborn, Germany \\ (Received 11 July 1996; revised manuscript received 4 November 1996)
}

\begin{abstract}
The results of electrical-resistivity $\rho$ measurements $(1.4-300 \mathrm{~K})$ on the alloys, $\mathrm{Ce}_{1-x} \mathrm{La}_{x} \mathrm{Ir}_{2} \mathrm{Ge}_{2}$ $(0 \leqslant x \leqslant 1), \mathrm{CeIr}_{2-x}(\mathrm{Rh}, \mathrm{Pt})_{x} \mathrm{Ge}_{2}(x=0.2$ and 0.4$)$, and $\mathrm{CeIr}_{2} \mathrm{Ge}_{2-x}(\mathrm{Si}, \mathrm{Sn})_{x}(x=0.2$ and 0.4$)$, are reported in order to understand the Kondo effect in $\mathrm{CeIr}_{2} \mathrm{Ge}_{2}$. There is a significant decrease in $\rho$ as the temperature is lowered from 100 to $4.2 \mathrm{~K}$ for $x=0.0$. This feature disappears for a small replacement of Ce by La $(x=0.3)$, thereby resulting in a single-ion Kondo effect for higher values of $x$. This finding establishes that the temperature $T_{\text {coh }}$, characterizing the coherent scattering among the Kondo centers for $x=0$ is as large as about $100 \mathrm{~K}$. The coherent scattering is not destroyed by small substitutions at the Ir or Ge site. The observed sensitivity of this coherence effect to a small disruption of Ce sublattice periodicity alone by La substitution is uncommon among trivalent Ce alloys. Such a large $T_{\text {coh }}$ value enables us to emphasize the need to invoke three characteristic temperatures for nonmagnetic Kondo lattices. [S0163-1829(97)04806-6]
\end{abstract}

Depending upon the degree of $4 f$ hybridization with the conduction band, the nature of the ground state of $\mathrm{Ce}$ and other physical characteristics have been found to vary from one compound to the other. As a result, the interest in $\mathrm{Ce}$ compounds remains unabated. It is a well-known fact $^{1-3}$ that, in many Kondo lattices, the coherent scattering among the Kondo centers as the temperature is lowered results in a marked drop of electrical resistance $(\rho)$ below a characteristic temperature $\left(T_{\text {coh }}\right)$. Most of the Ce compounds, in which $\mathrm{Ce}$ is nearly trivalent (that is, weakly hybridized systems), are characterized by low $T_{\text {coh }}$ values (less than $20 \mathrm{~K}$ ). Generally, one sees Fermi-liquid characteristics below a certain temperature $T_{\text {coh }}^{*}$, which is lower than $T_{\text {coh }}$. Several years ago, Anderson ${ }^{4}$ and Coleman ${ }^{5}$ put forward the idea that the temperature, $T_{\text {coh }}^{*}$, below which the quasiparticle bands arise, differ from the single-ion spin-fluctuation temperature by a factor of $1 /(2 j+1$ ) (where $j=5 / 2$ for fully degenerate trivalent $\mathrm{Ce}$ ion). This idea was subsequently discussed to describe the $\rho$ behavior of the mixed-valent compound $\mathrm{CePd}_{3}$ by Lawrence et al. ${ }^{6}$ However there were no further experimental reports aimed at probing whether the nonmagnetic Kondo lattices, particularly the alloys containing trivalent $\mathrm{Ce}$ ions, are characterized by multiple characteristic energies, viz., $T_{\text {coh }}^{*} T_{\text {coh }}$ and $T_{K}$ (where $T_{K}$ is the single-ion Kondo temperature). One of the reasons for this situation could be that the value of $T_{\text {coh }}$ is generally well below $20 \mathrm{~K}$ in most of the trivalent $\mathrm{Ce}$ alloys and hence the temperature window available to address this question is rather limited. Therefore, there is a need to identify trivalent Ce compounds in which the onset of the coherence effect is pushed to higher temperatures. In this respect, the recently discovered CeFeGe 3 (Refs. 7-10) in which $T_{\text {coh }}$ is about $100 \mathrm{~K}$ is of significance. In this article, we present the results of $\rho$ measurements on a nonmagnetic $\mathrm{Ce}$ compound, viz., $\mathrm{CeIr}_{2} \mathrm{Ge}_{2}$, (Refs. 11-13) and some of its pseudoternary alloys. The results establish the onset of Kondo coherent scattering near
$100 \mathrm{~K}$ in this compound. Our results suggest that these three characteristic energies are widely different in this compound and, apparently, there is no simple relationship between $T_{\text {coh }}^{*}$ and $T_{K}$. The other observations to be emphasized are (i) The amount of isovalent ion (viz., La) required to suppress the coherence peak in $\rho$ is unusually small for a trivalent $\mathrm{Ce}$ compound with such a large value of $T_{\text {coh }}$ and (ii) $\rho$ exhibits quasilinear dependence on temperature below about $11 \mathrm{~K}$ (which is referred to as $T_{\mathrm{coh}}^{*}$ as far as this compound is concerned in this article) rather than the quadratic dependence.

The samples, $\mathrm{Ce}_{1-x} \mathrm{La}_{x} \mathrm{Ir}_{2} \mathrm{Ge}_{2}(x=0.0,0.1,0.2,0.3,0.5$, 0.7, 0.9, and 1.0), $\operatorname{CeIr}_{2-x}(\mathrm{Rh}, \mathrm{Pt})_{x} \mathrm{Ge}_{2}(x=0.2$ and 0.4$)$ and $\mathrm{CeIr}_{2} \mathrm{Ge}_{2-x}(\mathrm{Si}, \mathrm{Sn})_{x}(\mathrm{x}=0.2$ and 0.4$)$, were prepared by arc melting. Some of these alloys were subjected to homogenization in evacuated sealed quartz tubes at $800{ }^{\circ} \mathrm{C}$ for week and we noticed that the resistivity of the homogenized ingots is significantly higher than that of the as-cast specimens. This implies that homogenization creates crystallographic imperfections and hence we restricted our studies reported here to as-cast specimens. The samples were characterized by $\mathrm{x}$-ray diffraction and the lines could be indexed on the basis of $\mathrm{CaBe}_{2} \mathrm{Ge}_{2}$-type tetragonal structure. The $\rho$ measurements were performed $(1.4-300 \mathrm{~K})$ by a conventional four-probe method using a silver paint for making electrical contacts; due to microcracks in the samples, no comparison of the values of $\rho$ may be made among these alloys. Ce $L_{\mathrm{III}}$-edge x-ray-absorption measurements for $\mathrm{CeIr}_{2} \mathrm{Ge}_{2}$ were performed at selected temperatures between 10 and $300 \mathrm{~K}$ at the EXAFS-II beamline of HASYLAB (DESY, Hamburg).

The results of $\rho$ measurements normalized to respective $300 \mathrm{~K}$ values are shown in Fig. 1 . In the case of $\mathrm{CeIr}_{2} \mathrm{Ge}_{2}$, there is a small increase of $\rho$ with decreasing temperature $(T)$ down to about $100 \mathrm{~K}$, following which there is a gradual fall from about $0.39 \mathrm{~m} \Omega \mathrm{cm}$ at $100 \mathrm{~K}$ to about $0.23 \mathrm{~m} \Omega \mathrm{cm}$ at $2 \mathrm{~K}$. Thus there is a reduction of $\rho$ by about $40 \%$. It may be remarked that Mathur and Frost ${ }^{12}$ have reported a very 


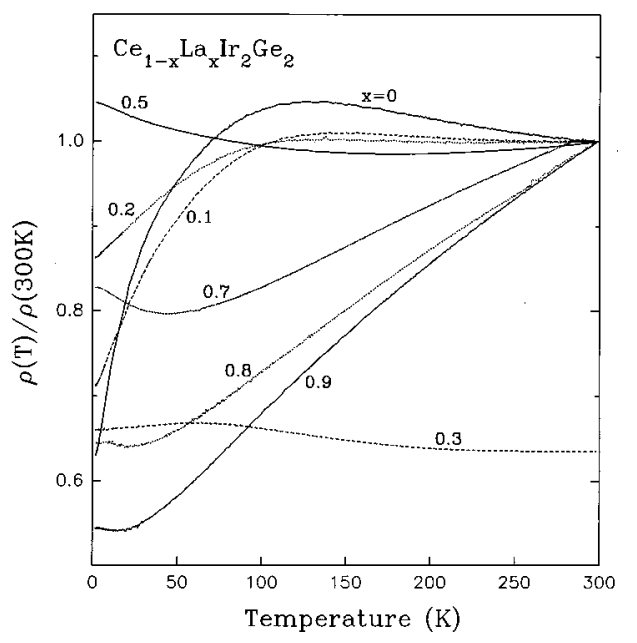

FIG. 1. The electrical resistivity $(\rho)$ as a function of temperature $(T)$ in the alloys, $\mathrm{Ce}_{1-x} \mathrm{La}_{x} \mathrm{Ir}_{2} \mathrm{Ge}_{2}$, normalized to respective $300 \mathrm{~K}$ values. The data for $x=0.3$ is shifted down on the vertical axis for the sake of clarity.

small value of 0.05 for $\rho(4.2 \mathrm{~K}) / \rho(300 \mathrm{~K})$ on the best platelets of this compound. The enhancement of this ratio in our alloy (to about 0.6 ) is presumably due to strong grainboundary effects in the way we have prepared these alloys. For this reason, no significance may be attached to absolute values of this ratio in all our specimens, though qualitative conclusions drawn on the basis of the trends in these values from one composition to the other are still valid. Such large drops of $\rho$ below $100 \mathrm{~K}$ cannot be explained in terms of crystal-field effects and we have therefore proposed the existence of Kondo coherence effects around $100 \mathrm{~K}$ (Ref. 13). In order to establish this interpretation, one should look for the features in the $\rho$ data when the Ce sublattice is diluted with a nonmagnetic rare-earth ion. Thus the $\rho$ data of Lasubstituted alloys shown in Fig. 1 are conclusive. For $x=0.1, \rho$ is nearly independent of temperature above $100 \mathrm{~K}$ and it falls by about $27 \%$ as the temperature is lowered to 2 $\mathrm{K}$; for $x=0.2$, the corresponding drop is about $15 \%$. For $x=0.3$, the low-temperature drop is suppressed and the decrease in $\rho$ is less than $1 \%$ below $60 \mathrm{~K} ; d \rho / d T$ is negative in the temperature range 60-300 K; these features are characteristic of single-ion Kondo effect. Further substitution of La, viz., $x=0.5$, results in complete modification of the overall features and the low temperature drop is no longer observed, instead there is a broad minimum around $180 \mathrm{~K}$ with a rise of about $6 \%$ from 180 to $2 \mathrm{~K}$. For $x=0.7$, the lattice contribution dominates above $50 \mathrm{~K}$, resulting in the Kondo minimum at about $45 \mathrm{~K}$; the percentage increase in low temperature $\rho$ is less than $3 \%$. For $x=0.8$, the overall features are the same, except that the minimum is shifted to $30 \mathrm{~K}$ and the magnitude of the low-temperature rise is around $1 \%$. For $x=0.9$, the Kondo minimum is apparently absent, as, following the composition dependence of the percentage increase of low-temperature rise, the expected upturn should be extremely small.

From the above experimental observations, it is clear that the destruction of the periodicity of the Ce sublattice by the substitution of La suppresses the drop of $\rho$ below $100 \mathrm{~K}$. This suppression of the low-temperature fall with La substi-

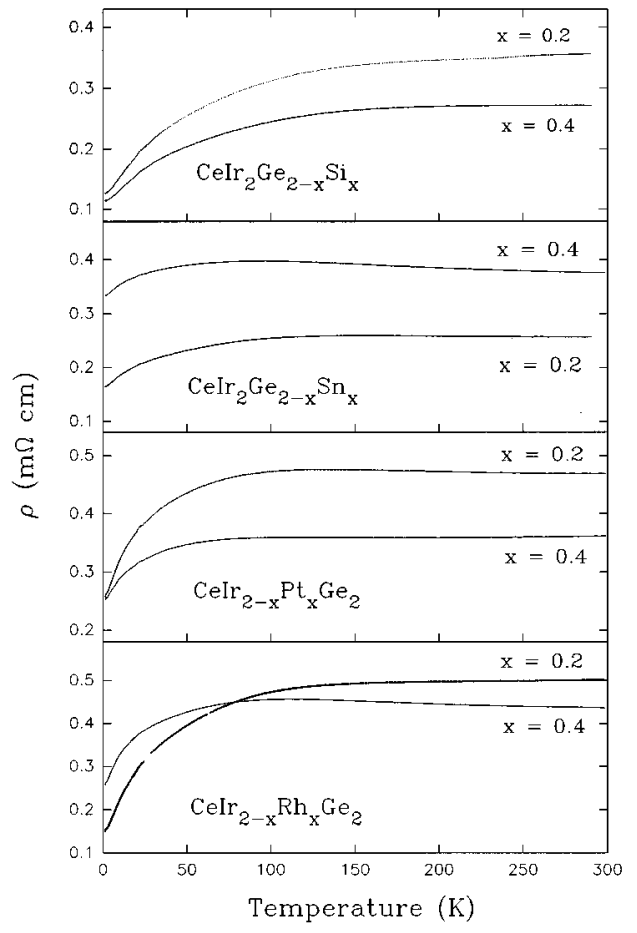

FIG. 2. The electrical resistivity as a function of temperature for several alloys of $\mathrm{CeIr}_{2} \mathrm{Ge}_{2}$ substituted for $\mathrm{Ir}$ and $\mathrm{Ge}$.

tution can be explained only if one assumes that this drop arises from the coherent scattering among the Kondo centers. Such a situation involving a disruption of the Ce periodicity by a small $(x=0.3)$ isovalent ionic substitution is not frequently met with in a Ce-based Kondo lattice with such a large Kondo coherence temperature in the nearly trivalent limit of Ce. Even in the series, $\mathrm{Ce}_{1-x} \mathrm{La}_{x} \mathrm{FeGe}_{3}$, a larger concentration of $\mathrm{La}(x=0.5)$ is required to destroy coherence effects ${ }^{9,10}$ and, in this sense, the behavior of $\mathrm{CeIr}_{2} \mathrm{Ge}_{2}$ appears to be unusual. We have also probed the sensitivity of the coherence-derived peak in $\rho$ to small substitutions at the non-Ce site (Rh or Pt for Ir; Si or Sn for Ge), the results of which are shown in Fig. 2. It is to be noted that this feature persists in all these alloys. In fact, initial substitutions $(x=0.2)$ of $\mathrm{Rh}$ and Si result in a drop by $70 \%$ and of Pt by about $45 \%$, thus making the coherence effect in fact more prominent; further increase of $x$ to 0.4 appears to change $\rho(T) / \rho(300 \mathrm{~K})$ towards the original value. Sn substitution apparently does not significantly alter a coherence-related drop of $\rho$. From these observations, it can be concluded that the chemical pressure, both positive or negative, involving isoelectronic as well as nonisoelectronic substitutions at the $\mathrm{Ir} / \mathrm{Ge}$ site does not result in the destruction of the coherence effect. The substitutional effect at the Ce site by $\mathrm{La}$, to the contrary, establishes that the periodicity of the Ce sublattice is crucial for the coherence effect at such a high temperature.

It may be recalled that the coherence effect around $100 \mathrm{~K}$ has been known ${ }^{6}$ in mixed-valent $\mathrm{CePd}_{3}$, in which case a distinct deviation of the lattice constants from the lanthanide contraction characteristic of the intermediate valence for $\mathrm{Ce}$ is well known. In contrast, in $\mathrm{CeIr}_{2} \mathrm{Ge}_{2}$, the lattice constants follow ${ }^{11}$ lanthanide contraction at room temperature as if $\mathrm{Ce}$ is more trivalent in this compound compared to $\mathrm{CePd}_{3}$. In order to address this question in a straightforward manner, 


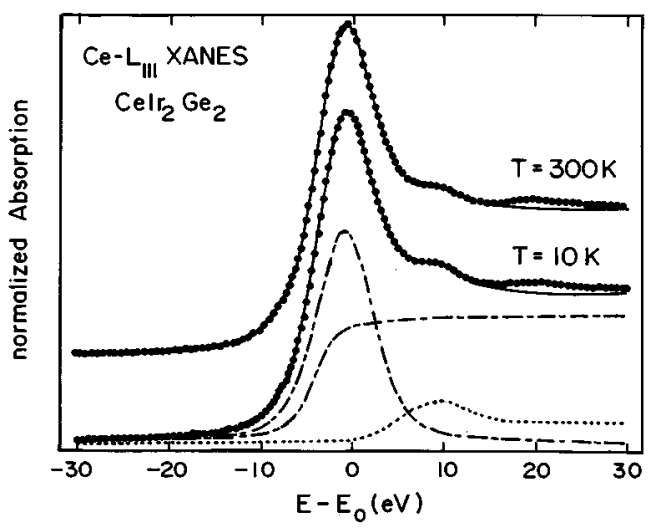

FIG. 3. Ce $L_{\mathrm{III}}$-edge spectra of $\mathrm{CeIr}_{2} \mathrm{Ge}_{2}$ at 300 and $10 \mathrm{~K}$. For $\mathrm{Ce}^{3+}$, the white line (a Lorentzian) and the edge (an arctan function) are also shown by long-dashed lines. The $\mathrm{Ce}^{4+}$ subspectrum (short-dashed) shown is the sum of these two components. $E_{0}$ corresponds to the white line of a reference $\left(\mathrm{CeF}_{3}\right)$.

we show the Ce $L_{\mathrm{III}}$-edge spectra of $\mathrm{CeIr}_{2} \mathrm{Ge}_{2}$ at 10 and 300 $\mathrm{K}$ in Fig. 3. In this spectroscopy, one observes a pronounced absorption peak (white line) at the edge position corresponding to a $2 p_{3 / 2}$ to $5 d$ transition. In metallic Ce systems with tetravalent admixture, one observes a double-peaked edge profile one each from $\mathrm{Ce}^{3+}$ and $\mathrm{Ce}^{4+}$ in the initial state, shifted by nearly $10 \mathrm{eV}$. The valency is generally derived from the relative amount of $\mathrm{Ce}^{3+}$ and $\mathrm{Ce}^{4+}$ edge profiles. In the present system, there is a prominent peak near $E_{0}$, defined by the maximum absorption (white line) of the $\mathrm{CeF}_{3}$ reference at $5725 \mathrm{eV}$ and a small tetravalent component, shifted by about $10 \mathrm{eV}$ to higher energy. From an usual line-shape analysis, ${ }^{14}$ we derive that the mean valence of $\mathrm{Ce}$ is $3.07 \pm 0.02$ and $3.1 \pm 0.02$ at 300 and $10 \mathrm{~K}$, respectively. Thus these results establish that $\mathrm{Ce}$ is close to trivalency in this compound down to $10 \mathrm{~K}$.

It is also of interest to compare the absolute values of $\mathrm{Ce}$ valency in $\mathrm{CePd}_{3}$ and $\mathrm{CeIr}_{2} \mathrm{Ge}_{2}$. A visual comparison of the spectra of $\mathrm{CePd}_{3}$ (Ref. 15) suggests that the tetravalent component in $\mathrm{CePd}_{3}$ is comparatively higher. The heat-capacity $(C)$ and magnetic-susceptibility $(\chi)$ results $^{13}$ are also consistent with these conclusions. The value of the linear term ( $\gamma$ ) obtained from the plot of $C / T$ versus $T^{2}$ below $20 \mathrm{~K}$ is about $130 \mathrm{~mJ} / \mathrm{mol} \mathrm{K} \mathrm{K}^{2}$ for $\mathrm{CeIr}_{2} \mathrm{Ge}_{2}$, which may be compared with $40 \mathrm{~mJ} / \mathrm{mol} \mathrm{K}^{2}$ for $\mathrm{CePd}_{3}$ (Ref. 16). The relatively large value for the former suggests that the $4 f$ orbital of Ce in the Ir sample is more localized compared with that in $\mathrm{CePd}_{3}$. From the $4 f$ contribution $\left(\chi_{4 f}\right)$ to $\chi$, obtained after subtracting $\chi(300 \mathrm{~K})$ of $\mathrm{LaIr}_{2} \mathrm{Ge}_{2}$, the values of $\mu_{\text {eff }}$ and $\theta_{p}$ for $x=0.0$ are found to be $2.5 \mu_{\beta}$ and $-160 \mathrm{~K}$, respectively, from the linear region of the plot of inverse $\chi$ versus $T$ above $200 \mathrm{~K}$. The value of the effective moment proves that $\mathrm{Ce}$ ions are trivalent.

We now pay some attention to an observation in the lowtemperature $\rho$ data. We notice that $\rho$ at low temperatures exhibits a quasilinear behavior in $\mathrm{CeIr}_{2} \mathrm{Ge}_{2}$, instead of the expected quadratic behavior (see Fig. 4). The lowtemperature $\rho$ data are plotted for three compositions in two different ways in Fig. 4 to highlight this point. It appears that $\rho$ varies with $T^{1.2}$ over a wide temperature range (up to 11 $\mathrm{K})$, in contrast to near-quadratic behavior only till $4 \mathrm{~K}$. The

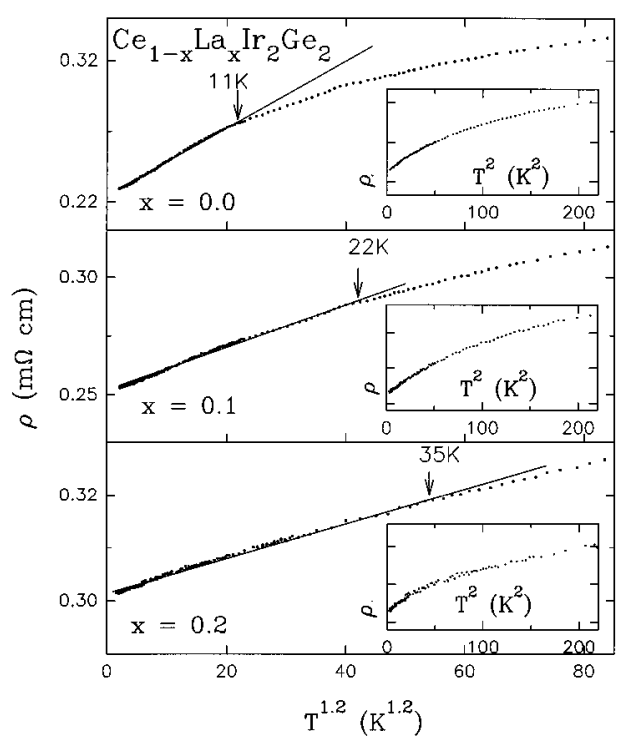

FIG. 4. The electrical resistivity $(\rho)$ versus $T^{1.2}$ at low temperatures for three alloys of the series, $\mathrm{Ce}_{1-x} \mathrm{La}_{x} \mathrm{Ir}_{2} \mathrm{Ge}_{2}$. In the insets, the data at lower temperatures are plotted as a function of the square of temperature. The continuous lines drawn through the data points show the linear region.

range of quasilinear behavior extends to about 22 and $35 \mathrm{~K}$ for $x=0.1$ and 0.2 , respectively, and apparently this feature persists (see Fig. 1) even in the Kondo-impurity regime, e.g., $x=0.5$, up to at least $30 \mathrm{~K}$. We also attempted to subtract the lattice contribution employing the values of $\mathrm{LaIr}_{2} \mathrm{Ge}_{2}$ and we noted that the quasilinear variation extends to a few more degrees Kelvin. We, however, do not show the $4 f$ contribution to $\rho$ thus obtained in the form of plots, as there is no systematics in the absolute values of $\rho$ as stated earlier. It can be confidently stated that a non-Fermi-liquid behavior ${ }^{17}$ in $\rho$ is demonstrated in a Kondo-lattice system with a high $T_{\text {coh. }}$. The low-temperature divergence (non-Fermi-liquid behavior $)^{17,18}$ in $C$ is however not noted $;{ }^{10}$ instead $C / T$ varies linearly with $T^{2}$ below $20 \mathrm{~K}$ as mentioned above. Though one is tempted to attribute the observed anomaly in $\rho$ to small intrinsic crystallographic disorder possibly giving rise to a distribution ${ }^{19}$ in the single-ion Kondo temperature, the trends seen in the alloys substituted at $\mathrm{Ir} / \mathrm{Ge}$ sites as argued below do not appear to support this idea. The $T^{1.2}$ dependence of $\rho$ gets modified with the substitutions at Ge and Ir sites; below $7 \mathrm{~K}, \mathrm{Si}$ and $\mathrm{Rh}$ substitutions restore $T^{2}$ dependence, while $\mathrm{Pt}$ and $\mathrm{Sn}$ substitutions result in $T^{1.5}$ dependence, thereby showing a tendency towards Fermi-liquid behavior; since these chemical substitutions naturally result in additional crystallographic disorder possibly resulting in a distribution of the $T_{K}$ value, one should have observed more deviations from Fermi-liquid behavior in these substituted alloys. The findings to the contrary in the substituted alloys therefore suggest that the distribution in $T_{K}$ resulting from possible crystallographic imperfections may not be the true cause of the anomaly in $\mathrm{CeIr}_{2} \mathrm{Ge}_{2}$. At this juncture, it is to be noted that the low-temperature $T^{1.2}$ behavior observed ${ }^{20}$ in the high-pressure phase of $\mathrm{CePd}_{2} \mathrm{Si}_{2}$ is being reproduced without the application of an external pressure in $\mathrm{CeIr}_{2} \mathrm{Ge}_{2}$. Further systematic studies, extended to the millikelvin range on several substituted samples, are needed to throw more 
light on the ground state, in view of the fact that the highpressure phase of $\mathrm{CePd}_{2} \mathrm{Si}_{2}$ undergoes heavy fermion superconducting transition around $0.5 \mathrm{~K}^{20}$

From the above data, it is clear that there is a temperature $\left(T_{\text {coh }}^{*}\right)$ below which one sees non-Fermi-liquid behavior and that the value of $T_{\text {coh }}^{*}$ is far below $T_{\text {coh }}$. It is of interest to compare the values of $T_{\text {coh }}^{*}$ and $T_{\text {coh }}$ with that of $T_{K}$. As mentioned in this paper, the value of $T_{K}$ given by $\theta_{p}$ is large. One can obtain ${ }^{8,21}$ a more precise value of $T_{K}$ from that of $\chi$ at $2 \mathrm{~K}$ and it turns out to be $185 \mathrm{~K}$. This value also agrees with that determined ${ }^{21}$ from $\gamma$ data $\left(T_{K}=180 \mathrm{~K}\right)$. While determining the value of $T_{K}$ from $\chi(2 \mathrm{~K})$ and $\gamma$ data, we have assumed $j=5 / 2$ in the relationship between these quantities. ${ }^{8,21}$ It is clear that the value of $T_{K}$ is significantly larger than those of $T_{\mathrm{coh}}^{*}$ and $T_{\mathrm{coh}}$. This finding implies that this Kondo lattice is characterized by three energies, $T_{\text {coh }}^{*}$, $T_{\text {coh }}$, and $T_{K}$. This conclusion is qualitatively consistent with the ideas discussed in Refs. 4-6. Quantitatively, the value of $T_{\text {coh }}^{*}$ for $\mathrm{CeIr}_{2} \mathrm{Ge}_{2}$ is less than half of the expected value, that is, $T_{K} / 2 j+1$. If one strictly defines $T_{\text {coh }}^{*}$ as a temperature until which $\rho$ exhibits quadratic temperature dependence (in which case $T_{\mathrm{coh}}^{*}<4 \mathrm{~K}$ ), then the deviation from the expected value is clearly still larger. This observation supports the opinion of Lawrence et al. ${ }^{6}$ that the two energies $T_{\mathrm{coh}}^{*}$ and $T_{K}$ are not uniquely related and that the relationship appears to depend on various other details like crystal structure, band structure, etc.

To conclude, in the compound $\mathrm{CeIr}_{2} \mathrm{Ge}_{2}$, the onset of Kondo coherent scattering effect occurs at a rather high temperature (close to $100 \mathrm{~K}$ ). This effect could be destroyed by an unusually small isovalent ionic substitution at the Ce site. Unlike other Kondo lattices, $\rho$ apparently exhibits nonFermi-liquid behavior below about $11 \mathrm{~K}$. The large value of $T_{\text {coh }}$ enables us to revive the idea ${ }^{6}$ of invoking multiple characteristic energies even in those Kondo lattices in which $\mathrm{Ce}$ valency is close to 3 .
${ }^{1}$ See, for instance, N. B. Brandt and V. V. Moshchalkov, Adv. Phys. 33, 373 (1984).

${ }^{2}$ U. Rauchschwalbe, Physica B 147, 1 (1987).

${ }^{3}$ J. D. Thompson, J. M. Lawrence, and Z. Fisk, J. Low Temp. Phys. 95, 59 (1994).

${ }^{4} \mathrm{P}$. W. Anderson, in Valence Fluctuations in Solids, edited by L. M. Falicov, W. Hanke, and M. B. Maple (North-Holland, Amsterdam, 1981), p. 451.

${ }^{5}$ P. Coleman, Phys. Rev. B 28, 5255 (1983).

${ }^{6}$ J. M. Lawrence, J. D. Thompson, and Y. Y. Chen, Phys. Rev. Lett. 54, 2537 (1985); J. M. Lawrence, Y. Y. Chen, and J. D. Thompson, in Theoretical and Experimental Aspects of Valence Fluctuations and Heavy Fermions, edited by L. C. Gupta and S. K. Malik (Pergamon, New York, 1987), p. 169, and references therein; J. M. Lawrence, T. Graf, M. F. Hundley, D. Mandrus, J. D. Thompson, A. Lacerda, M. S. Torikachvili, J. L. Sarrao, and Z. Fisk, Phys. Rev. B 53, 12559 (1996).

${ }^{7}$ H. Yamamoto, H. Sawa, and M. Ishikawa, Phys. Lett. A 196, 83 (1994).

${ }^{8}$ H. Yamamoto, M. Ishikawa, K. Hasegawa, and J. Sakurai, Phys. Rev. B 52, 10136 (1995).

${ }^{9}$ H. Yamamoto and M. Ishikawa, J. Phys. Soc. Jpn. 65, 50 (1995).

${ }^{10}$ E. V. Sampathkumaran and I. Das, Physica B 223-224, 310 (1996).

${ }^{11}$ M. Francois, G. Venturini, J. F. Mareche, B. Malaman, and B. Roques, J. Less Common Met. 113, 231 (1985).
${ }^{12}$ N. D. Mathur and C. D. Frost, J. Alloys Compd. 215, 325 (1994).

${ }^{13}$ E. V. Sampathkumaran and R. Mallik, Physica B 223-224, 316 (1996).

${ }^{14}$ E. V. Sampathkumaran, K. H. Frank, G. Kalkowski, G. Kaindl, M. Domke, and G. Wortmann, Phys. Rev. B 29, 5702 (1984), and references therein.

${ }^{15}$ S. Raaen, M. L. den Boer, V. Murgai, and R. D. Parks, Phys. Rev. B 27, 5139 (1983); R. D. Parks, S. Raaen, M. L. den Boer, V. Murgai, and T. Mihalisin, ibid. 28, 3556 (1983).

${ }^{16}$ R. Selim, D. Michels, and T. Mihalisin, J. Magn. Magn. Mater. 47-48, 99 (101).

${ }^{17}$ M. B. Maple, C. L. Seaman, D. A. Gajewski, Y. Dalichaouch, V. B. Barbetta, M. C. de Andrade, H. A. Mook, H. G. Lukefahr, O. O. Bernal, and D. E. MacLaughlin, J. Low Temp. Phys. 95, 225 (1994), and references therein; H. v. Löhneysen, M. Sieck, O. Stockert, and M. Waffenschmidt, Physica B 223-224, 471 (1996); M. B. Maple, D. A. Gajewski, R. Chau, P. Dai, H. A. Mook, R. Movshovich, and C. L. Seaman, ibid. 223-224, 447 (1996), and references therein.

${ }^{18}$ P. D. Sacramento and P. Schlottmann, Phys. Rev. B 43, 13294 (1991).

${ }^{19}$ V. Dobrosavljevic, T. R. Kirkpatrick, and G. Kotliar, Phys. Rev. Lett. 69, 1113 (1992).

${ }^{20}$ F. M. Grosche, S. R. Julian, N. D. Mathur, and G. G. Lonzarich, Physica B 223-224, 50 (1996).

${ }^{21}$ B. Coqblin and J. R. Schrieffer, Phys. Rev. 185, 847 (1969); V. T. Rajan, Phys. Rev. Lett. 51, 308 (1983). 